\title{
Decomposition of Hyper Spaces of Soft Sets
}

\author{
Alkan ÖZKAN $^{1}$
}

ABSTRACT: In this paper, firstly, we define two new hyperspaces of soft sets called co-compact and co-Lindelöf topological spaces and obtain some properties of them. Secondly, by use these topological spaces we define the C-continuous and L-continuous of soft multifunction and obtain some characterization of them. Finally, we give the decomposition of these topological spaces with Vietoris soft topological spaces.

Keywords: Soft continuity, soft hyperspaces, soft multifunction, soft sets, soft topological spaces

\section{Esnek Kümelerin Hiper Uzaylarının Ayrıştırılması}

ÖZET: Bu makalede ilk olarak, esnek ko-kompakt ve esnek ko-Lindelöf topolojik uzaylar olarak isimlendirilen esnek kümelerde iki yeni hiper uzay tanımladık ve bazı özelliklerini elde ettik. İkinci olarak, bu uzayları kullanarak esnek küme değerli dönüşümlerin C-sürekliliği ve L-sürekliğini tanımladık ve bazı karakterizasyonlarını elde ettik. Son olarak, bu iki uzayın birbiriyle ve bir diğer hiper uzay olan Vietoris topolojik uzay ile karşılaştırmasını verdik.

Anahtar kelimeler: Esnek hiper uzay, esnek küme, esnek küme değerli dönüşüm, esnek süreklilik, esnek topolojik uzay

Iğdır Üniversitesi, Fen Edebiyat Fakültesi, Matematik Bilgisayar, Iğdır, Türkiye

eSorumlu yazar/Corresponding Author: Alkan ÖZKAN, alkan.ozkan@igdir.edu.tr 


\section{INTRODUCTION}

Soft set theory has rich potential for practical applications in several sciences. Molodtsov (1999) introduced the concept of soft sets. Pei and Miao (2005) investigated the relationships between soft sets and information systems. Çağman et al. (2011) defined a soft topological space. Zorlutuna et al. (2012) studied some concepts in soft topological spaces. Çağman and et al. (2011) redefined the operations of the soft sets and constructed a uni-int decision making method by using these new operations. Then Akdağ and Erol $(2014,2015)$ introduced the concept of soft multifunction and studied their properties in many paper. Many researchers studied on soft set theory such as Shabir and Naz (2011), Weijian (2012), Varol and et al. (2012), Roy and Samanta (2014), Nazmul and Samanta (2013).

\section{MATERIALS AND METHODS \\ Preliminaries}

Definition 2.1. Let $S(X, E)$ be the family of all soft sets and $(U, E)$ be a soft open set in $S(X, E)$. The soft set families $(U, E)^{+}$and $(U, E)^{-}$are defined as follows:

$(U, E)^{+}=\{(G, E) \in S(X, E):(T, E) \widetilde{\subset}(U, E)\}$,

$(U, E)^{-}=\{(G, E) \in S(X, E):(T, E) \widetilde{\cap}(U, E) \neq \Phi\}$. (Akdağ et al., 2015-b)

Proposition 2.2. For non-null soft sets $(G, E)$ and $(H, E)$ in $S(X, E)$ the following statements are true;

(a) $(G, E)^{+} \cap(H, E)^{+}=((G, E) \widetilde{\cap}(H, E))^{+}$

(b) $(G, E)^{+} \cup(H, E)^{+} \subset((G, E) \widetilde{\cup}(H, E))^{+}$

(c) $((G, E) \widetilde{\cap}(H, E))^{-} \subset(G, E)^{-} \cap(H, E)^{-}$

(d) $(G, E)^{-} \cup(H, E)^{-}=((G, E) \widetilde{\cup}(H, E))^{-}$

(e) $(G, E) \widetilde{\subset}(H, E)$ if and only if $(G, E)^{+} \subset(H, E)^{+}$

(f) $(G, E) \widetilde{\subset}(H, E)$ if and only if $(G, E)^{-} \subset(H, E)^{-}$

(g) $(G, E) \widetilde{\cap}(H, E)=\Phi$ if and only if $(G, E)^{+} \cap(H, E)^{+}=\Phi$

(h) $(G, E)^{-} \cap(H, E)^{-} \neq \Phi$. (Akdağ et al., 2015-b)

Proposition 2.3. Let $(X, \tau, E)$ be a soft topological space and $\mathbb{S}(X, E)$ be the family of all null soft sets. Then the soft set families

$$
\begin{aligned}
& \beta_{1}=\left\{(U, E)^{+}:(U, E) \text { soft open set }\right\} \\
& \beta_{2}=\left\{(U, E)^{-}:(U, E) \text { soft open set }\right\}
\end{aligned}
$$

are soft base (soft sub base) for different soft topological spaces on $\mathbb{S}(X, E)$, resp. (Akdağ et al., 2015-b)

Definition 2.4. The soft topological spaces which mentioned in above proposition are called

soft upper Vietoris and soft lower Vietoris and denoted by $\tau_{S V^{+}}, \tau_{S V^{-}}$, respectively.

The family of soft sets $\beta_{1} \cup \beta_{2}=\left\{(U, E)^{+},(U, E)^{-}:(U, E)\right.$ soft open set $\}$ is the soft sub base for a soft topological space. This space is called Vietoris soft topological space and denoted by $\tau_{S V}$. Also, $\tau_{S V}{ }^{+} U \tau_{S V}{ }^{-}$is soft base for the soft vietoris topology. (Akdağ et al., 2015-b)

Definition 2.5. Let $S(X, E)$ and $S(Y, K)$ be two families of soft sets. Let $u: X \rightarrow Y$ be multifunction and $p: E \rightarrow K$ be mapping. Then a soft multifunction $F: S(X, E) \rightarrow S(Y, K)$ is defined as follows:

For a soft set $(G, A)$ in $S(X, E),(F(G, A), K)$ is a soft set in $(Y, K)$ given by

$$
F(G, A)(k)= \begin{cases}\bigcup_{e \in p^{-1}(k) \cap A} u(G(e)) & \text {, if } p^{-1}(k) \cap A \neq \emptyset \\ \varnothing & \text {,if } p^{-1}(k) \cap A \neq \emptyset\end{cases}
$$

$(F(G, A), K)$ is called soft image of a soft set $(G, A)$.

Moreover, $F(G, A)=\widetilde{U}\left\{F\left(E_{e}{ }^{x}\right): E_{e}{ }^{x} \widetilde{\in}(G, A)\right\}$. (Akdağ et al., 2015-a)

Definition 2.6. Let $F: S(X, E) \rightarrow S(Y, K)$ be a soft multifunction.

i. The soft upper inverse image of $(H, K)$ denoted by $F^{+}(H, K)$ and defined as

$F^{+}(H, K)=\left\{E_{e}{ }^{x} \widetilde{\in} \tilde{X}: F\left(E_{e}{ }^{x}\right) \widetilde{\subset}(H, K)\right\}$

ii. The soft lower inverse image of $(H, K)$ denoted by $F^{-}(H, K)$ and defined as

$F^{-}(H, K)=\left\{E_{e}{ }^{x} \widetilde{\in} \tilde{X}: F\left(E_{e}{ }^{x}\right) \widetilde{\cap}(H, K) \neq \Phi\right\}$.

Also, $F(\tilde{X})=\widetilde{U} F\left(E_{e}{ }^{x}\right)$. (Akdağ et al., 2015-a)

Definition 2.7. Let $F, G: X \rightarrow Y$ be two soft multifunctions. For $E_{e}{ }^{x} \widetilde{\in} \tilde{X}$, the combination and intersection of $F$ and $G$ is denoted by

$(F \cup G)\left(E_{e}{ }^{x}\right)=F\left(E_{e}{ }^{x}\right) \widetilde{\cup} G\left(E_{e}{ }^{x}\right)$, 
$(F \cap G)\left(E_{e}{ }^{x}\right)=F\left(E_{e}{ }^{x}\right) \widetilde{\cap} G\left(E_{e}{ }^{x}\right)$ resp. (Akdağ et al., 2015-b)

Definition 2.8. Let $F: S(X, E) \rightarrow S(Y, K)$ and $G: S(X, E) \rightarrow S(Y, K)$ be two soft multifunctions. Then, $F$ equal to $G$ if $F\left(E_{e}{ }^{x}\right)=G\left(E_{e}{ }^{x}\right)$, for each $E_{e}{ }^{x} \widetilde{\in} \tilde{X}$. (Akdağ et al., 2015-a)

Definition 2.9. The soft multifunction $F: S(X, E) \rightarrow S(Y, K)$ is called surjective if $p$ and $u$ are surjective. (Akdağ et al., 2015-a)

Theorem 2.10. Let $F: S(X, E) \rightarrow S(Y, K)$ be a soft multifunction. For soft sets $(F, E),(G, E)$ and for a family of soft sets $\left(G_{i}, E\right)_{i \in I}$ in soft family $S(X, E)$ the following are hold:
(a) $F(\Phi)=\Phi$
(b) $F(\tilde{X}) \widetilde{\subset} \tilde{Y}$
(c) $F((G, A) \widetilde{\cup}(H, B))=F(G, A) \widetilde{\cup} F(H, B)$ and in general
$F\left(\widetilde{\mathrm{U}}_{i}\left(G_{i}, E\right)\right)=\widetilde{\mathrm{U}}_{i} F\left(G_{i}, E\right)$
(d) $F((G, A) \widetilde{\cap}(H, B)) \widetilde{\subset} F(G, A) \widetilde{\cap} F(H, B)$ in general
$F\left(\cap_{i}\left(G_{i}, E\right)\right) \widetilde{\subset} \widetilde{\cap}_{i} F\left(G_{i}, E\right)$

(e) If $(G, E) \widetilde{\subset}(H, E)$, then $F(G, E) \widetilde{\subset} F(H, E)$. (Akdağ et al., 2015-a)

Theorem 2.11. Let $F: S(X, E) \rightarrow S(Y, K)$ be a soft multifunction. For soft sets $(G, K),(H, K)$ in soft family $S(Y, K)$ the following statements are true:
(a) $F^{-}(\Phi)=\Phi$ and $F^{+}(\Phi)=\Phi$
(b) $F^{-}(\tilde{Y})=\tilde{X}$ and $F^{+}(\widetilde{Y})=\tilde{X}$
(c) $F^{-}((G, K) \widetilde{\cup}(H, K))=F^{-}(G, K) \widetilde{\cup} F^{-}(H, K)$
(d) $F^{+}(G, K) \widetilde{\cup} F^{+}(H, K) \widetilde{\subset} F^{+}((G, K) \widetilde{\cup}(H, K))$
(e) $F^{-}((G, K) \widetilde{\cap}(H, K)) \widetilde{\subset} F^{-}(G, K) \widetilde{\cap} F^{-}(H, K)$
(f) $F^{+}(G, K) \widetilde{\cap} F^{+}(H, K)=F^{+}((G, K) \widetilde{\cap}(H, K))$
(g) If $(G, K) \widetilde{\subset}(H, K)$, then $F^{-}(G, K) \widetilde{\subset} F^{-}(H, K)$ and $F^{+}(G, K) \widetilde{\subset} F^{+}(H, K)$. (Akdağ et al., 2015-a)

Proposition 2.12. Let $F: S(X, E) \rightarrow S(Y, K)$ be a soft multifunction. For a soft subset $(G, E)$ in $X$ and for soft subsets $(H, K),(U, K)$ in $Y$ the following statements are true:

(a) $(G, E) \widetilde{\subset} F^{+}(F(G, E)) \widetilde{\subset} F^{-}(F(G, E))$. If $F$ is surjectice then

$(G, E)=F^{+}(F(G, E))=F^{-}(F(G, E))$

(b) $F\left(F^{+}(H, K)\right) \widetilde{\subset}(H, K) \widetilde{\subset} F\left(F^{-}(H, K)\right)$.

(c) If $(H, K) \widetilde{\cap}(U, K)=\Phi$ then $F^{+}(H, K) \widetilde{\cap} F^{-}(U, K)=\Phi$. (Akdağ et al., 2015-a)

Proposition 2.13. Let $F:(X, \tau, E) \rightarrow(Y, \sigma, K)$ and $G:(Y, \sigma, K) \rightarrow(Z, \eta, L)$ be soft multifunction. Then the followings are true;

(a) $\left(F^{-}\right)^{-}=F$

(b) $(G o F)^{-}(T, C)=F^{-}\left(G^{-}(T, C)\right)$ and $(G o F)^{+}(T, C)=F^{+}\left(G^{+}(T, C)\right)$, for a soft subset $(T, C)$ in $Z$. (Akdağ et al., 2015-a)

Proposition 2.14. Let $F, G:(X, \tau, E) \rightarrow(Y, \sigma, K)$ be two soft multifunctions. For a soft set $(H, K)$ in $Y$ the following statements are hold:
(a) $(F \cup G)^{-}(H, K)=F^{-}(H, K) \widetilde{\cup} G^{-}(H, K)$.
(b) $(F \cup G)^{+}(H, K)=F^{+}(H, K) \widetilde{\cup} G^{+}(H, K)$
(c) $(F \cap G)^{-}(H, K) \widetilde{\subset} F^{-}(H, K) \widetilde{\cap} G^{-}(H, K)$
(d) $F^{+}(H, K) \widetilde{\cap} G^{+}(H, K) \widetilde{\subset}(F \cap G)^{+}(H, K)$. (Akdağ et al., 2015-b)

Proposition 2.15. Let $F:(X, \tau, E) \rightarrow(Y, \sigma, K)$ be soft multifunction. The followings are true for soft set $(G, K)$ in $Y$;

(a) $F^{+}(\tilde{Y}-(G, K))=\tilde{X}-F^{-}(G, K)$

(b) $F^{-}(\tilde{Y}-(G, K))=\tilde{X}-F^{+}(G, K)$. (Akdağ et al., 2015-a)

Definition 2.16. A soft topological space $(X, \tau, E)$ is called soft compact space if each open soft cover of $\tilde{X}$ has a finite soft subcover. (Zorlutuna et al., 2012)

Definition 2.17. A soft topological space $(X, \tau, E)$ is called soft Lindelöf if each open soft cover of $\tilde{X}$ has a soft countable subcover. (Weijian, 2012)

Definition 2.18. A soft topological space $(X, \tau, E)$ is called soft Lindelöf closed if each Lindelöf soft set in it are soft closed.

Proposition 2.19. Let $(X, \tau, E)$ be a soft compact space and $(M, E)$ be a closed soft set, then $(M, E)$ is a soft compact set. (Varol et al., 2012)

Definition 2.20. Let $(X, \tau, E)$ be a soft topological space and $E_{e}{ }^{x}, E_{e}{ }^{y} \widetilde{\in} \tilde{X}$ such that $E_{e}{ }^{x} \neq E_{e}{ }^{y}$. Then $(X, \tau, E)$ 
is called a soft Hausdorff space if there exist open soft sets $(F, E)$ and $(G, E)$ such that $E_{e}^{x} \widetilde{\in}(F, E), E_{e}{ }^{y} \widetilde{\in}(G, E)$ and $(F, E) \widetilde{\cap}(G, E)=\phi$. (Shabir et al., 2011)

\section{Upper (Lower) Co-Compact and Co-Lindelöf Topological Spaces}

Lemma 3.1. Let $(Y, \tau, K)$ be soft topological space. The followings are true for soft set $(G, K)$ in $Y$.

i) $S(Y, K)-(G, K)^{+}=(\tilde{Y}-(G, K))^{+}$

ii) $S(Y, K)-(G, K)^{-}=(\tilde{Y}-(G, K))$

Proof. i) $(B, K) \in S(Y, K)-(G, K)^{+} \Leftrightarrow(B, K) \notin(G, K)^{+} \Leftrightarrow(B, K) \widetilde{\not}(G, K)$

$\Leftrightarrow(B, K) \widetilde{\cap}(\tilde{Y}-(G, K)) \neq \phi \Leftrightarrow(B, K) \in(\tilde{Y}-(G, K))^{-}$.

ii) $(B, K) \in S(Y, K)-(G, K)^{-} \Leftrightarrow(B, K) \notin(G, K)^{-} \Leftrightarrow(B, K) \widetilde{\cap}(G, K)=\phi$

$\Leftrightarrow(B, K) \widetilde{\subset}(\tilde{Y}-(G, K)) \Leftrightarrow(B, K) \in(\tilde{Y}-(G, K))$.

Lemma 3.2 For soft sets $\left(C_{1}, K\right),\left(C_{2}, K\right)$ in $S(Y, K)$ the following statements are true:

i) $\left[S(Y, K)-\left(C_{1}, K\right)^{-}\right] \widetilde{\cap}\left[S(Y, K)-\left(C_{2}, K\right)^{-}\right]=S(Y, K)-\left[\left(C_{1}, K\right)^{-} \cup\left(C_{2}, K\right)^{-}\right]$

ii) $S(Y, K)-\left[\left(C_{1}, K\right)^{+} \cup\left(C_{2}, K\right)^{+}\right]=\left[S(Y, K)-\left(C_{1}, K\right)^{+}\right] \widetilde{\cap}\left[S(Y, K)-\left(C_{2}, K\right)^{+}\right]$

Proof. i) $(H, K) \in\left[S(Y, K)-\left(C_{1}, K\right)^{-}\right] \widetilde{\cap}\left[S(Y, K)-\left(C_{2}, K\right)^{-}\right]$

$\Leftrightarrow(H, K) \in S(Y, K)-\left(C_{1}, K\right)^{-}$and $(H, K) \in S(Y, K)-\left(C_{2}, K\right)^{-}$

$\Leftrightarrow(H, K) \notin\left(C_{1}, K\right)^{-}$and $(H, K) \notin\left(C_{2}, K\right)^{-}$

$\Leftrightarrow(H, K) \widetilde{\cap}\left(C_{1}, K\right)=\phi$ and $(H, K) \widetilde{\cap}\left(C_{2}, K\right)=\phi$

$\Leftrightarrow(H, K) \widetilde{\cap}\left[\left(C_{1}, K\right) \cup\left(C_{2}, K\right)\right]=\phi$

$\Leftrightarrow(H, K) \notin\left[\left(C_{1}, K\right) \cup\left(C_{2}, K\right)\right]^{-}$

$\Leftrightarrow(H, K) \in S(Y, K)-\left[\left(C_{1}, K\right) \cup\left(C_{2}, K\right)\right]^{-}$

$\Leftrightarrow(H, K) \in S(Y, K)-\left[\left(C_{1}, K\right)^{-} \cup\left(C_{2}, K\right)^{-}\right]$.

ii) $(H, K) \in S(Y, K)-\left[\left(C_{1}, K\right)^{+} \cup\left(C_{2}, K\right)^{+}\right]$

$\Leftrightarrow(H, K) \notin\left[\left(C_{1}, K\right)^{+} \cup\left(C_{2}, K\right)^{+}\right]$

$\Leftrightarrow(H, K) \notin\left(C_{1}, K\right)^{+}$and $(H, K) \notin\left(C_{2}, K\right)^{+}$

$\Leftrightarrow(H, K) \in S(Y, K)-\left(C_{1}, K\right)^{+}$and $(H, K) \in S(Y, K)-\left(C_{2}, K\right)^{+}$

$\Leftrightarrow(H, K) \in\left[S(Y, K)-\left(C_{1}, K\right)^{+}\right] \widetilde{\cap}\left[S(Y, K)-\left(C_{2}, K\right)^{+}\right]$.

Proposition 3.3. Let $(Y, \tau, K)$ be a soft topological space. Then the soft set families

$$
\begin{gathered}
\beta_{3}=\left\{S(Y, K)-(C, K)^{-}:(C, K) \text { compact }\right\} \widetilde{\cup}\{\phi\} \\
\beta_{4}=\left\{S(Y, K)-(C, K)^{+}:(C, K) \text { compact }\right\}
\end{gathered}
$$

are soft base and soft subbase for different soft topological spaces on $S(Y, K)$, respectively.

Proof. We know that $(C, K)=\phi$ is soft compact set and $(C, K)^{-}=\emptyset$. Therefore $S(Y, K)-(C, K)^{-}=$ $S(Y, K) \in \beta_{3}$. Thus $S(Y, K)=\mathrm{U}_{(B, K) \in \beta_{3}}(B, K)$. For $S(Y, K)-\left(C_{1}, K\right)^{-}, S(Y, K)-\left(C_{2}, K\right)^{-} \in \beta_{3}$ let $(H, K) \in$ $\left[S(Y, K)-\left(C_{1}, K\right)^{-}\right] \widetilde{\cap}\left[S(Y, K)-\left(C_{2}, K\right)^{-}\right]$. Since

$\left[S(Y, K)-\left(C_{1}, K\right)^{-}\right] \widetilde{\cap}\left[S(Y, K)-\left(C_{2}, K\right)^{-}\right]=S(Y, K)-\left[\left(C_{1}, K\right)^{-} \cup\left(C_{2}, K\right)^{-}\right] \quad$ then $\quad(H, K) \in S(Y, K)-$ $\left[\left(C_{1}, K\right)^{+} \cup\left(C_{2}, K\right)^{+}\right]$. Also we have $\left(C_{1}, K\right)^{-} \cup\left(C_{2}, K\right)^{-}=\left(\left(C_{1}, K\right) \widetilde{\cup}\left(C_{2}, K\right)\right)^{-}$. Since $\left(C_{1}, K\right) \widetilde{\cup}\left(C_{2}, K\right)$ is soft compact set then $S(Y, K)-\left[\left(C_{1}, K\right)^{-} \cup\left(C_{2}, K\right)^{-}\right] \in \beta_{3}$. Thus $\beta_{3}$ is soft base for a soft topological space.

Similarly it can be show that $\beta_{4}$ is soft sub base for a soft topological space.

Definition 3.4. The soft topological spaces which mentioned in above proposition are called soft upper cocompact and soft lower co-compact and denoted by $\tau_{S C^{+}}, \tau_{S C^{-}}$, respectively.

Lemma 3.5. A soft closed set in a soft compact topological spaces is soft compact.

Proof. Let $(X, \tau, E)$ be a soft compact topological spaces and $(H, E)$ be a soft closed set in $X$. Let the family $\left\{\left(G_{i}, E\right): i \in I\right\}$ be a soft open cover of $(H, E)$. Then the family $\left\{\left(G_{i}, E\right): i \in I\right\} \widetilde{U}(\tilde{X}-(H, E))$ a soft open cover of $\tilde{X}$. Since $(X, \tau, E)$ is a soft compact topological spaces then we obtain $\left\{\left(G_{i_{1}}, E\right), \ldots,\left(G_{i_{n}}, E\right)\right\} \widetilde{\sim}(\tilde{X}-(H, E))$ is a finite soft cover of $\tilde{X}$. Therefore $\left\{\left(G_{i_{1}}, E\right), \ldots,\left(G_{i_{n}}, E\right)\right\}$ is a finite soft cover of $(H, E)$ and thus $(H, E)$ is soft compact.

Lemma 3.6. A soft compact set in a soft Hausdorff topological spaces is soft closed.

Proof. Let $(X, \tau, E)$ be a soft Hausdorff topological spaces and $(C, E)$ be a soft compact set in $X$. We will show that $\tilde{X}-(C, E)$ is soft open set. Let $E_{e}{ }^{x} \widetilde{\in} \tilde{X}-(C, E)$ then $E_{e}{ }^{x} \widetilde{\notin}(C, E)$. Since $(X, \tau, E)$ is soft Hausdorff topological spaces, for every soft point $E_{e}{ }^{y} \widetilde{\in}(C, E)$ such that $E_{e}{ }^{x} \neq E_{e}{ }^{y}$, there exists $\left(U_{y}, E\right)$ and $\left(P_{y}, E\right)$ two soft neighborhood of $E_{e}{ }^{x}, E_{e}{ }^{y}$ resp., such that $\left(U_{y}, E\right) \widetilde{\cap}\left(P_{y}, E\right)=\phi$. Then the family $\left\{\left(P_{y}, E\right): E_{e}{ }^{y} \widetilde{\in}(C, E)\right\}$ is the soft open neighborhood of $(C, E)$. Since $(C, E)$ is soft compact set there exists a finite soft sub cover such that $(C, E) \widetilde{\subset} \cup_{i=1}^{n}\left(P_{y_{i}}, E\right)$. Since $\left(U_{y_{i}}, E\right) \widetilde{\cap}\left(P_{y_{\dot{\gamma}}}, E\right)=\phi$ for every $y_{i} \in\left\{y_{1}, \ldots, y_{n}\right\}$ then we have that $\left(\mathrm{U}_{i=1}^{n}\left(U_{y_{i}}, E\right)\right) \tilde{\cap}\left(P_{y_{i}}, E\right)=\phi$. Also $U_{i=1}^{n}\left(U_{y_{i}}, E\right)$ is a soft neighborhood of soft point $E_{e}{ }^{x}$. 
$\left(\bigcup_{i=1}^{n}\left(U_{y_{i}}, E\right)\right) \widetilde{n}\left(\bigcup_{j=1}^{n}\left(P_{y^{\prime}}, E\right)\right)=\phi$ for $y_{i} \in\left\{y_{1}, \ldots, y_{n}\right\}$. So, $\left(\bigcup_{i=1}^{n}\left(U_{y_{i}}, E\right)\right) \widetilde{n}(C, E)=\phi$ and thus we obtain $E_{e}{ }^{x} \tilde{\in}\left(\bigcup_{i=1}^{n}\left(U_{y_{i}}, E\right)\right) \widetilde{C} \tilde{X}-(C, E)$. Therefore $\tilde{X}-(C, E)$ is soft open set and $(C, E)$ is soft closed set.

Remark 3.7. In a compact and Hausdorff soft topological spaces, a soft set is compact if and only if it soft closed.

Theorem 3.8. Let $(Y, \tau, K)$ be a soft topological space. Then the following statements are true for the soft topological spaces $\tau_{S C^{+}}, \tau_{S C^{-}}, \tau_{S V^{+}}, \tau_{S V^{-}}$on $S(Y, K)$ :

i) If $(Y, \tau, K)$ Hausdorff then $\tau_{S C^{+}} \leq \tau_{S V^{+}}$and $\tau_{S C^{-}} \leq \tau_{S V^{-}}$.

ii) If $(Y, \tau, K)$ Hausdorff and compact then $\tau_{S C^{+}}=\tau_{S V^{+}}$and $\tau_{S C^{-}}=\tau_{S V^{-}}$.

Proof. i) Let $S(Y, K)-(C, K)^{-} \in \beta_{3}$. Since $S(Y, K)-(C, K)^{-}=(\tilde{Y}-(C, K))^{+},(C, K)$ is soft compact and $(Y, \tau, K)$ Hausdorff then $\tilde{Y}-(C, K)$ is a soft open set. Then $(\tilde{Y}-(C, K))^{-} \in \tau_{S V^{+}}$and $S(Y, K)-(C, K)^{-} \in \tau_{S V^{+}}$. Thus we have that $\tau_{S C^{+}} \leq \tau_{S V^{+}}$.

Similarly by use $S(Y, K)-(C, K)^{+}=(\tilde{Y}-(C, K))^{-}$it can be show that $\tau_{S C^{-}} \leq \tau_{S V^{-}}$.

ii) In a Hausdorff and compact soft topological space, the compact soft sets are soft closed and the soft closed sets are soft compact. Therefore we have $\tau_{S C^{+}}=\tau_{S V^{+}}$and $\tau_{S C^{-}}=\tau_{S V^{-}}$.

Definition 3.9. Let $(X, \vartheta, E),(Y, \tau, K)$ be two soft topological spaces and $E_{e}{ }^{x_{0}}$ be soft point in $X$. Then the soft multifunction $F:(X, \vartheta, E) \rightarrow(Y, \tau, K)$ is called;

i) soft upper $C$ continuous at $E_{e}{ }^{x_{0}}$ if for each soft compact set $(C, K)$ such that $F\left(E_{e}{ }^{x_{0}}\right) \widetilde{\cap}(C, K)=\phi$, there exists $(P, E)$ soft neighbourhood of $E_{e}{ }^{x_{0}}$ such that $F\left(E_{e}{ }^{x}\right) \widetilde{\cap}(C, K)=\phi$ for every $E_{e}{ }^{x} \widetilde{\in}(P, E)$.

ii) soft lower $C$ continuous at $E_{e}{ }^{x_{0}}$ if for each soft set $(C, K)$ such that $F\left(E_{e}{ }^{x_{0}}\right) \widetilde{\cap}(C, K) \neq \phi$ and $\tilde{Y}-(C, K)$ is soft compact, there exists $(P, E)$ a soft neighbourhood of $E_{e}{ }^{x_{0}}$ such that $F\left(E_{e}{ }^{x}\right) \widetilde{\cap}(C, K) \neq \phi$ for every $E_{e}^{x} \widetilde{\in}(P, E)$.

\section{RESULTS AND DISCUSSION}

Theorem 3.10. Let $F:(X, \vartheta, E) \rightarrow(Y, \tau, K)$ be soft multifunction. Then the following statements are true;

i) $F$ is soft upper $\mathrm{C}$ continuous at $E_{e}{ }^{x_{0}}$ if and only if the mapping $f:(X, \vartheta, E) \rightarrow\left(S(Y, K), \tau_{S C^{+}}, K\right)$ is continuous at $E_{e}{ }^{x_{0}}$.

ii) $F$ is soft lower $C$ continuous at $E_{e}{ }^{x_{0}}$ if and only if $f:(X, \vartheta, E) \rightarrow\left(S(Y, K), \tau_{S C^{-}}, K\right)$ is continuous at $E_{e}{ }^{x_{0}}$.

Proof. i) $(\Rightarrow)$ Let $f\left(E_{e}^{x_{0}}\right) \in S(Y, K)-(C, K)^{-} \in \beta_{3}$. Then we have that $f\left(E_{e}{ }^{x_{0}}\right) \in(\tilde{Y}-(C, K))^{+}=$ $\{(A, K):(A, K) \widetilde{\subset} \widetilde{Y}-(C, K)\}$ and $f\left(E_{e}{ }^{x_{0}}\right) \widetilde{\subset} \tilde{Y}-(C, K)$. Then $f\left(E_{e}{ }^{x_{0}}\right) \widetilde{\cap}(C, K)=\phi$ and $F\left(E_{e}{ }^{x_{0}}\right) \widetilde{\cap}(C, K)=\phi$. Since $F$ is soft upper $C$ continuous at $E_{e}{ }^{x_{0}}$, then there exists $(P, E)$ a soft neighbourhood of $E_{e}{ }^{x_{0}}$ such that $F\left(E_{e}{ }^{x}\right) \widetilde{\cap}(C, K)=\phi$ for every $E_{e}{ }^{x} \in(P, E)$. Thus $f\left(E_{e}{ }^{x}\right) \notin(C, K)^{-}$and $f\left(E_{e}{ }^{x}\right) \in S(Y, K)-(C, K)^{-}$. Thus we have that

$f:(X, \vartheta, E) \rightarrow\left(S(Y, K), \tau_{S C^{+}}, K\right)$ is continuous at $E_{e}{ }^{x_{0}}$.

$(\Leftarrow)$ Let $(C, K)$ be a soft compact set such that $F\left(E_{e}{ }^{x_{0}}\right) \widetilde{\cap}(C, K)=\phi$. Then

$f\left(E_{e}{ }^{x_{0}}\right) \notin(C, K)^{-} \quad$ and $f\left(E_{e}{ }^{x_{0}}\right) \in S(Y, K)-(C, K)^{-} \in \beta_{3}$. Since $f:(X, \vartheta, E) \rightarrow\left(S(Y, K), \tau_{S C^{+}}, K\right)$ is continuous at $E_{e}{ }^{x_{0}}$, then there exists $(P, E)$ a soft neighbourhood of $E_{e}{ }^{x_{0}}$ such that $f(P, E) \in S(Y, K)-(C, K)^{-}$. Thus $f\left(E_{e}{ }^{x}\right) \in S(Y, K)-(C, K)^{-}$for every $E_{e}{ }^{x} \in(P, E)$. Therefore $F\left(E_{e}{ }^{x}\right) \widetilde{\cap}(C, K)=\phi$. Hence $F$ is soft upper $\mathrm{C}$ continuous at $E_{e}{ }^{x_{0}}$.

ii) Similarly it can be proof.

Theorem 3.11. Let $(Y, \tau, K)$ be a Hausdorff soft topological spaces. Then the following statements are equivalent for a soft multifunction $F:(X, \vartheta, E) \rightarrow(Y, \tau, K)$.

i) $F$ is soft upper $C$ continuous .

ii) $F^{+}(V, K)$ is soft open set for every soft open set $(V, K)$ such that $\tilde{Y}-(V, K)$ is soft compact.

iii) $F^{-}(H, K)$ is soft closed set for every soft compact set $(H, K)$.

Proof. (i $\Rightarrow$ ii) Let $E_{e}{ }^{x_{0}} \in F^{+}(V, K)$ and $(V, K)$ be a soft open set such that $\tilde{Y}-(V, K)$ is soft compact. Then $F\left(E_{e}{ }^{x_{0}}\right) \widetilde{\subset}(V, K)$ and $F\left(E_{e}{ }^{x_{0}}\right) \widetilde{\cap}(\tilde{Y}-(V, K)) \neq \phi$. Since $F$ is soft upper $C$ continuous at $E_{e}{ }^{x_{0}}$, there exists $(P, E)$ a soft neighbourhood of $E_{e}{ }^{x_{0}}$ such that $F\left(E_{e}{ }^{x}\right) \widetilde{\subset}(V, K)$ for every soft point $E_{e}{ }^{x} \in(P, E)$. Then we obtain $E_{e}{ }^{x_{0}} \in(P, E) \widetilde{\subset} F^{+}(V, K)$ and $E_{e}{ }^{x_{0}} \in \operatorname{int}\left(F^{+}(V, K)\right)$. Therefore $F^{+}(V, K) \widetilde{i n t}\left(F^{+}(V, K)\right)$. This shows that $F^{+}(V, K)$ is soft open set.

(ii $\Rightarrow$ iii) Let $(H, K)$ be a soft compact set. Then $(H, K)$ is soft closed. So, $\tilde{Y}-(H, K)$ is soft open set such that its complement is soft compact. By hypothesis, $F^{+}(\tilde{Y}-(H, K))$ is soft open. Since $F^{+}(\tilde{Y}-(H, K))=\tilde{X}-$ $\left(F^{-}(H, K)\right)$, then $\tilde{X}-\left(F^{-}(H, K)\right)$ is soft open and $F^{-}(H, K)$ soft closed . 
(iii $\Rightarrow$ i) Let $(C, K)$ be soft compact set with $F\left(E_{e}{ }^{x_{0}}\right) \widetilde{\cap}(C, K)=\phi$. Then $F\left(E_{e}{ }^{x_{0}}\right) \widetilde{\subset} \tilde{Y}-(C, K)$. By hypthesis $F^{-}(C, K)$ is soft closed and thus $\tilde{X}-\left(F^{-}(C, K)\right)=F^{+}(\tilde{Y}-(C, K))$ is soft open and $E_{e}{ }^{x_{0}} \in F^{+}(\tilde{Y}-(C, K))$. If we take $(P, E)=F^{+}(\tilde{Y}-(C, K))$ soft open neighborhood of $E_{e}{ }^{x_{0}}$, then we obtain $F\left(E_{e}{ }^{x}\right) \widetilde{\subset} \tilde{Y}-(C, K)$ for every $E_{e}{ }^{x} \in F^{+}(\tilde{Y}-(C, K))$ and thus $F\left(E_{e}{ }^{x}\right) \widetilde{\cap}(C, K)=\phi$. Hence $F$ is soft upper $C$ continuous at $E_{e}{ }^{x_{0}}$.

Theorem 3.12. Let $F:(X, \vartheta, E) \rightarrow(Y, \tau, K)$ be a soft multifunction. The following statements are equivalent;

i) $F$ is soft lower $C$ continuous

ii) $F^{-}(V, K)$ is soft open set for every soft open set $(V, K)$ such that $\tilde{Y}-(V, K)$ is compact.

iii) $F^{+}(H, K)$ is soft closed set for every soft compact set $(H, K)$.

Proof. Similar to previous proposition it can be proof.

Proposition 3.13. Let $(Y, \tau, K)$ be a soft topological space. Then the soft set families

$$
\begin{gathered}
\beta_{5}=\left\{S(Y, K)-(L, K)^{+}:(L, K) \text { Lindelö } f\right\} \widetilde{\cup}\{\phi\} \\
\beta_{6}=\left\{S(Y, K)-(L, K)^{-}:(L, K) \text { Lindelö } f\right\}
\end{gathered}
$$

are soft base and soft sub base for a different soft topological spaces on $S(Y, K)$, respectively.

Proof. Since $(L, K)=\phi$ is soft Lindelöf set and $(L, K)^{+}=\emptyset$ then $S(Y, K)-(L, K)^{+}=S(Y, K) \in \beta_{5}$. Thus $S(Y, K)=\mathrm{U}_{(B, K) \in \beta_{5}}(B, K)$.

Let $(H, K) \in S(Y, K)-\left(L_{1}, K\right)^{+}$and $(H, K) \in S(Y, K)-\left(L_{2}, K\right)^{+}$. Then we have that

$(H, K) \in\left[S(Y, K)-\left(L_{1}, K\right)^{+}\right] \widetilde{\cap}\left[S(Y, K)-\left(L_{2}, K\right)^{+}\right]=S(Y, K)-\left[\left(L_{1}, K\right)^{+} \cup\left(L_{2}, K\right)^{+}\right]$. Thus $\beta_{5}$ is soft base for a soft topological space.

Similarly it can be show that $\beta_{6}$ is soft sub base for a soft topological space.

Definition 3.14. The topological spaces which mentioned in above proposition are called soft upper coLindelöf and soft lower co-Lindelöf and denoted by $\tau_{S L^{+}}, \tau_{S L^{-}}$, respectively.

Lemma 3.15. Every soft compact set is soft Lindelöf set.

Proof. It is obvious from the definition of soft compact sets ans soft Lindelöf sets.

Definition 3.16. $(Y, \tau, K)$ is called Lindelöf-closed soft topological space if every soft Lindelöf set in $(Y, \tau, K)$ is soft closed set.

Theorem 3.17. Let $(Y, \tau, K)$ be a soft topological space. Then the following statements are true for the soft topological spaces $\tau_{S L^{+}}, \tau_{S L^{-}}, \tau_{S C^{+}}, \tau_{S C^{-}}, \tau_{S V^{+}}, \tau_{S V^{-}}$on $S(Y, K)$;

i) If $(Y, \tau, K)$ Lindelöf-closed soft topological space then $\tau_{S L^{+}} \leq \tau_{S V^{+}}$and $\tau_{S L^{-}} \leq \tau_{S V^{-}}$.

ii) $\tau_{S C^{+}} \leq \tau_{S L^{+}}$and $\tau_{S C^{-}} \leq \tau_{S L^{-}}$.

Proof. i) Let $S(Y, K)-(L, K)^{-} \in \beta_{5}$. Since $(L, K)_{+}$is Lindelöf soft set by hypothesis $(L, K)$ is soft closed. Also, we know that $S(Y, K)-(L, K)^{-}=(\tilde{Y}-(L, K))^{+}$and $(\tilde{Y}-(L, K)) \in \tau_{S V^{+}}$then we have $S(Y, K)-$ $(L, K)^{-} \in \tau_{S V^{+}}$. Thus $\tau_{S L^{+}} \leq \tau_{S V^{+}}$.

Similarly by use $S(Y, K)-(L, K)^{+}=(\tilde{Y}-(L, K))^{-}$it can be show that $\tau_{S L^{-}} \leq \tau_{S V^{-}}$

ii) In a soft topological space, the Lindelöf soft sets are soft compact. So, the proof is obvious.

Remark 3.18. Let $(Y, \tau, K)$ be a Lindelöf-closed soft topological space. Then

$\tau_{S C^{+}} \leq \tau_{S L^{+}} \leq \tau_{S V^{+}}$and $\tau_{S C^{-}} \leq \tau_{S L^{-}} \leq \tau_{S V^{-}}$.

Definition 3.19. Let $(X, \vartheta, E),(Y, \tau, K)$ be two soft topological spaces and $E_{e}{ }^{x_{0}}$ be soft point in $X$. The soft multifunction $F:(X, \vartheta, E) \rightarrow(Y, \tau, K)$ is called;

i) soft upper $L$ continuous at $E_{e}{ }^{x_{0}}$ if for each $(L, K)$ soft Lindelöf set with $F\left(E_{e}{ }^{x_{0}}\right) \widetilde{\cap}(L, K)=\phi$, there exists $(P, E)$ neighbourhood of $E_{e}{ }^{x_{0}}$ such that $F\left(E_{e}{ }^{x}\right) \widetilde{\cap}(L, K)=\phi$ for every $E_{e}{ }^{x} \widetilde{\in}(P, E)$.

ii) soft lower $L$ continuous at $E_{e}{ }^{x_{0}}$ if for each $(L, K)$ soft set with $F\left(E_{e}{ }^{x_{0}}\right) \widetilde{\cap}(L, K) \neq \phi$ and $\tilde{Y}-(L, K)$ is soft Lindelöf set, there exists $(P, E)$ soft neighbourhood of $E_{e}{ }^{x_{0}}$ such that $F\left(E_{e}{ }^{x}\right) \widetilde{\cap}(L, K) \neq \phi$ for every $E_{e}{ }^{x} \in(P, E)$.

Theorem 3.20. Let $F:(X, \vartheta, E) \rightarrow(Y, \tau, K)$ be a soft multifunction.

i) $F$ is soft upper $\mathrm{L}$ continuous at $E_{e}{ }^{x_{0}}$ if and only if the mapping $f:(X, \vartheta, E) \rightarrow\left(S(Y, K), \tau_{S L^{+}}, K\right)$ is continuous at $E_{e}{ }^{x_{0}}$.

ii) $F$ is soft lower L continuous at $E_{e}{ }^{x_{0}}$ if and only if $f:(X, \vartheta, E) \rightarrow\left(S(Y, K), \tau_{S L^{-}}, K\right)$ continuous at $E_{e}{ }^{x_{0}}$.

Proof. It can be show similar to Proposition 3.10.

Proposition 3.21. Let $(Y, \tau, K)$ be soft Lindelöf-closed topological space. Then the following statements are equivalent for a soft multifunction $F:(X, \vartheta, E) \rightarrow(Y, \tau, K)$;

i) $F$ is soft upper $\mathrm{L}$ continuous at $E_{e}{ }^{x_{0}}$ if and only if for every soft open set $(V, K)$ with $\tilde{Y}-(V, K)$ is soft Lindelöf and $F\left(E_{e}{ }^{x_{0}}\right) \widetilde{\subset}(V, K)$ there exists $(P, E)$ soft open neighborhood of $E_{e}{ }^{x_{0}}$ such that $F\left(E_{e}{ }^{x}\right) \widetilde{\subset}(V, K)$ for every $E_{e}{ }^{x} \widetilde{\in}(P, E)$.

ii) $F$ is soft lower $\mathrm{L}$ continuous at $E_{e}{ }^{x_{0}}$ if and only if for every soft open set $(V, K)$ with $\tilde{Y}-(V, K)$ is soft 
Lindelöf and $F\left(E_{e}{ }^{x_{0}}\right) \widetilde{\cap}(V, K) \neq \phi$ there exists $(P, E)$ soft open neighborhood of $E_{e}{ }^{x_{0}}$ such that $F\left(E_{e}{ }^{x}\right) \widetilde{\cap}(V, K) \neq \phi$ for every $E_{e}{ }^{x} \widetilde{\in}(P, E)$.

Proof. It can be show easily.

Theorem 3.22. Let $(Y, \tau, K)$ be soft Lindelöf-closed topological space. Then the following statements are equivalent for a soft multifunction $F:(X, \vartheta, E) \rightarrow(Y, \tau, K)$;

i) $F$ is soft upper $\mathrm{L}$ continuous if and only if $F^{+}(V, K)$ is soft open set for every soft open set $(V, K)$ with $\tilde{Y}-(V, K)$ is soft Lindelöf.

ii) $F$ is soft lower $\mathrm{L}$ continuous if and only if $F^{-}(V, K)$ is soft open set for every soft open set $(V, K)$ with $\tilde{Y}-(V, K)$ is soft Lindelöf.

Proof. i) $\quad \Leftrightarrow$ Let $\tilde{Y}-(V, K)$ be a soft Lindelöf set and $E_{e}{ }^{x_{0}} \in F^{+}(V, K)$. Then we have $F\left(E_{e}{ }^{x_{0}}\right) \widetilde{\subset} F\left(F^{+}(V, K)\right) \widetilde{\subset}(V, K)$ and thus $F\left(E_{e}{ }^{x_{0}}\right) \widetilde{\cap}(\tilde{Y}-(V, K))=\phi$. Since $\tilde{Y}-(V, K)$ soft Lindelöf and $F$ is soft upper L continuous at $E_{e}{ }^{x_{0}}$ then there exists $(P, E)$ soft open neighborhood of $E_{e}{ }^{x_{0}}$ such that $F(P, E) \widetilde{\cap}(\tilde{Y}-$ $(V, K))=\phi$. Then $F(P, E) \widetilde{\subset}(V, K)$ and thus $E_{e}{ }^{x_{0}} \in(P, E) \widetilde{\subset} F^{+}(V, K)$. Hence we have that $E_{e}{ }^{x_{0}} \in \operatorname{int}\left(F^{+}(V, K)\right)$. Thus $F^{+}(V, K) \widetilde{\sim} \operatorname{int}\left(F^{+}(V, K)\right)$ and $F^{+}(V, K)$ is soft open set.

$(\Leftarrow)$ Let $(L, K)$ be soft Lindelöf set and $F\left(E_{e}{ }^{x_{0}}\right) \widetilde{\cap}(L, K)=\phi$. Then $F\left(E_{e}{ }^{x_{0}}\right) \widetilde{\subset} \tilde{Y}-(L, K)$. Since $(Y, \tau, K)$ is soft Lindelöf-closed, by hypothesis we have $F^{+}(\tilde{Y}-(L, K))$ is soft open set and $E_{e}{ }^{x_{0}} \in F^{+}(\tilde{Y}-(L, K))$. Put $(P, E)=F^{+}(\tilde{Y}-(L, K))$ soft open neighborhood of $E_{e}{ }^{x_{0}}$. Thus $F\left(E_{e}{ }^{x_{0}}\right) \widetilde{\subset} F\left(F^{+}(\tilde{Y}-(L, K))\right) \widetilde{\subset}(\tilde{Y}-(L, K))$. Also $F\left(F^{+}(\widetilde{Y}-(L, K))\right) \widetilde{n}(L, K)=\phi$. Hence $F$ is soft upper L continuous at $E_{e}{ }^{x_{0}}$.

ii) $(\Rightarrow)$ Let $\tilde{Y}-(V, K)$ be soft Lindelöf set and $E_{e}{ }^{x_{0}} \in F^{-}(V, K)$. Then $F\left(E_{e}{ }^{x_{0}}\right) \widetilde{\cap}(V, K) \neq \phi$. Since $\tilde{Y}-(V, K)$ soft Lindelöf and $F$ is soft lower $\mathrm{L}$ continuous at $E_{e}{ }^{x_{0}}$ then there exists $(P, E)$ soft open neighborhood of $E_{e}{ }^{x_{0}}$ such that $F(P, E) \widetilde{\cap}(V, K)=\phi$. Then

$E_{e}{ }^{x_{0}} \in(P, E) \widetilde{\subset} F^{-}(V, K)$. Hence

$E_{e}{ }^{x_{0}} \in \operatorname{int}\left(F^{-}(V, K)\right)$. Thus we obtain

$F^{-}(V, K) \widetilde{\subset} \operatorname{int}\left(F^{-}(V, K)\right)$ and $F^{-}(V, K)$ is soft open set.

$(\Leftarrow)$ Let $\tilde{Y}-(V, K)$ be soft Lindelöf set and $F\left(E_{e}{ }^{x_{0}}\right) \widetilde{\cap}(V, K) \neq \phi$. Since $(Y, \tau, K)$ is soft Lindelöf-closed topological space then $(V, K)$ soft open set. By hypothesis $F^{-}(V, K)$ is soft open set and $E_{e}{ }^{x_{0}} \in F^{-}(V, K)$. Put $(P, E)=F^{-}(V, K)$ soft open neighborhood of $E_{e}{ }^{x_{0}}$. Thus we have that $F(P, E) \widetilde{\cap}(V, K) \neq \phi$. Hence $F$ is soft lower L continuous at $E_{e}{ }^{x_{0}}$.

\section{CONCLUSIONS}

In this paper we study on hyperspaces of soft sets. Then we give the relationship between of hyperspaces of soft sets. We obtain characterizations

\section{REFERENCES}

Akdağ M, Erol F, 2014. Upper and Lower Continuity of Soft Multifunctions, Appl. Math. Inf. Sci. 8, No. 6, 2873-2880.

Akdağ M, Erol F, 2015-a. Multifunction between soft topological spaces, International Journal of Mathematics Trends and Technology 20(1): 62-69.

Akdağ M, Erol F, 2015-b. On Hyperspaces of soft sets, Journal of New Theory, 7: 86-97.

Akdag M, Erol F, 2015-c. Soft b-continuous and soft b-irresolute multifunctions, Journal of Advanced Studies in Topology 6:3, 82-89.

Çağman N, Enginoğlu S, 2010. Soft set theory and uni-int decision making, European Journal of Operational Research, 207: 848855.

Çağman N, Karataş S, Enginoğlu S, 2011. Soft topology, Computer Math. Appl. 62: 351-358.

Molodtsov D, 1999. Soft set theory-First results, Comput. Math. with Appl. 37 (4/5): 19-31. of these hyper spaces and investigate properties. We expect that results in this paper will be basis for further applications of soft multifunctions in soft sets theory.

Nazmul SK and Samanta SK, 2013. Neighborhood properties of soft topological spaces, Annal. Fuzzy Math. Info. 6(1):1-15.

Pei D, Miao D, 2005. From soft sets to information systems, in Proceedings of the IEEE International Conference on Granular Computing, 2: 617-621.

Roy S, Samanta TK, 2014. A note on Soft Topological Spaces, Punjab University Journal of Mathematics, 46(1): 19-24.

Shabir M, Naz M, 2011. On soft topological spaces, Comput. Math. Appl. 61: 1786-1799.

Varol BP, Shostak A, Aygün H, 2012. A New Approach to Soft Topology, Hacettepe Journal of Mathematics and Statistics Volume 41(5): 731-74.

Weijian R, 2012. The countabilities of soft topological spaces, International Journal of Computational and Mathematical Sciences, 6: 159-162.

Zorlutuna I, Akdağ M, Min WK, Atmaca S, 2012. Remarks on soft topological spaces, Annals of Fuzzy Mathematics and Informatics, 3: 171-185. 